\title{
UMA NOVA PROPOSTA PARA A MODELAGEM DA PROPAGAÇÃO DE EPIDEMIAS
}

\author{
Alexandre R. N. Kulpel
}

\begin{abstract}
Resumo
Desde os primórdios da história a humanidade convive que doenças, muitas com um nível de letalidade e potencial epidêmico relativamente baixo, mas defrontando-se por vezes com epidemias que se desenvolvem com consequências catastróficas e ultrapassando até as barreiras continentais. Este artigo busca entender os mecanismos utilizados atualmente para modelar a propagação de epidemias ao longo da história mais recente e propor ferramentas e conceitos que permitam compreender, combater e prevenir a ocorrência de novos surtos epidêmicos.
\end{abstract}

Palavras-chave: modelagem, epidemias, redes

\begin{abstract}
Since the dawn of history humanity has coexisted with diseases, many with a relatively low level of mortality and epidemic potential, but sometimes being confronted with epidemies that develop with catastrophic consequences and even surpassing continental barriers. This article seeks to understand the mechanisms currently used to model the spread of epidemies throughout the most recent history and to propose tools and concepts to understand, combat and prevent the occurrence of new epidemic outbreaks.
\end{abstract}

Keywords: modeling, epidemics, nets 


\section{Introdução}

O mundo é um ambiente cada vez mais conectado, tanto no âmbito físico, quanto no virtual: estradas, rotas aéreas e náuticas tornam cidades, países e os continentes cada vez mais interligados e interconectados. Tão longe a humanidade caminhou neste sentido no último século, que passou a explorar até domínios fora do globo terrestre, exemplo disso é o anúncio recente da NASA a respeito da descoberta de corpos d'água no planeta Marte (NASA, 2015).

Além disso, cabos de fibra óptica, antenas de rádio e de celular, satélites e toda a infraestrutura de telecomunicações, criam uma camada complexa e abrangente de interligação virtual do mundo que, apesar de reforçar os modelos de centro-periferia (CASTELLS, 2013), fazem com que as distâncias sejam não apenas reduzidas, mas colocam o próprio conceito de distância em cheque (GRAHAN; MARVIN, 1997).

Este cenário de alta conectividade física e virtual tornou as sociedades e suas dinâmicas de interação cada vez mais complexas e, parafraseando Castells, cada vez mais em rede: redes de computadores, redes de informação, redes de telecomunicação, redes de abastecimento, redes de transporte, etc. (CASTELLS, 2013), não deixam dúvidas de que vivemos em um mundo estruturado e inteligível apenas à luz da dinâmica das redes.

O objetivo deste trabalho é mostrar alternativas para atualizar o ferramental utilizado na modelagem de epidemias, visando ampliar a capacidade de entendimento e predição da ocorrência de epidemias, para aperfeiçoar as ações tanto de contenção como, principalmente, de prevenção de epidemias.

Para isso, faremos (a) uma revisão sobre a modelagem de epidemias, mostrando as dificuldades de análise de populações estruturadas; (b) uma abordagem da modelagem através de redes da dinâmica de populações, com uma breve revisão da história da evolução dos modelos para o estudo das dinâmicas de propagação de epidemias, onde a importância de buscar formas mais abrangentes de análise fica evidente; (c) uma demonstração de como outras ferramentas mais complexas, como o uso de EDO's, do conceito de redes e de autômatos celulares podem viabilizar analises mais abrangentes e (d) apresentaremos nossas conclusões. 


\section{A modelagem de epidemias}

Segundo o dicionário Michaelis, uma epidemia é uma "doença que ataca ao mesmo tempo muitas pessoas da mesma terra ou região" ou "Ideias, sistemas ou coisas que se difundem com abundância e rapidez, dominando os espíritos ou os costumes" (MICHAELIS, 2012). Por definição é um fenômeno que afeta grandes quantidades de pessoas simultaneamente, de modo que há tempos a humanidade se preocupa em desenvolver ferramentas que lhe permitam compreender melhor a dinâmica de propagação das epidemias e buscar formas eficazes de combatê-las e, principalmente, evita-las.

Ao longo do século XX surgiram correntes de pensamento que objetivavam compreender e modelar a dinâmica de surgimento e propagação de epidemias, sendo que, em um primeiro momento, a matemática, com seus modelos quantitativos forneceu o corpo conceitual utilizado para estudar fenômenos epidêmicos. No entanto, os primeiros modelos quantitativos inspirados na Física limitavam-se ao estudo das populações como um todo, considerando-as completamente desprovidas de estrutura(s) interna(s) (CODEÇO; COELHO, 2008). Schimit também nos mostra a necessidade de ampliar horizonte de análise quando, ao falar sobre um modelo baseado em equações diferenciais afirma: "esse modelo, apesar de fornecer resultados e previsões importantes, apresenta limitações, sendo uma delas a consideração de que os indivíduos estão homogeneamente espalhados no espaço" (SCHIMIT, 2010, p. 15).

Posteriormente, na busca pela evolução do poder explicativo e qualidade dos modelos de propagação de epidemias, fez-se necessária a inclusão de fatores relativos à estrutura das populações, tais como: grupos sociais, redes sociais e coesão social (CODEÇO; COELHO, 2008). Surge, então, a necessidade de fazer com que os modelos dialoguem com a sociologia e outras ciências sociais, de modo que suas análises se beneficiem de uma visão mais ampla dos fatores envolvidos nos fenômenos sociais (ou influenciados pela estrutura social), e que, dado que a sociedade é o meio onde as interações humanas ocorrem, aceleram ou reduzem o ritmo de propagação das epidemias.

O conceito de redes teve, neste sentido, importante papel ao permitir um diálogo interdisciplinar que tanto enriquece os estudos de fenômenos sociais e/ou de outros fenômenos que também afetam as populações humanas, conforme fica claro no trecho a seguir:

O conceito de redes une várias disciplinas científicas no século XXI, dentre elas a epidemiologia, enquanto caminho para a busca de alternativas ao reducionismo presente na análise dos elementos 
desconectados do sistema, sem cair na contrapartida do obscurantismo

holista. É um olhar sobre as unidades e suas relações, as dependências entre os elementos, necessidade geral de todas as disciplinas, que precisam de formas de expressão simultânea da unidade, multiplicidade, totalidade, e organização dos sistemas complexos que estudam. (CODEÇO; COELHO, 2008, p. 1768)

Ao analisar a propagação de epidemias a partir de uma óptica que permita incorporar a lógica das redes sociais (no sentido de redes formadas no âmago da sociedade e pela conexão entre as diferentes sociedades) no estudo, passou-se de modelos que consideravam apenas dados demográficos agregados de uma população, como o número total de pessoas e sua distribuição no espaço (densidade e concentração demográfica), para modelos que melhor capturam a essência fluida e dinâmica das interações humanas. Ou seja, as interações e as formas de interação humanas, tão importantes na dinâmica das epidemias, passam também a compor os novos modelos desenvolvidos a partir desta lógica.

\section{História e evolução dos modelos de fenômenos epidêmicos}

É preciso reconstruir brevemente o caminho evolutivo percorrido pelas teorias e métodos utilizados para modelar epidemias que conduzem até o momento atual, onde o ferramental utilizado para estudar redes revelou-se de grande valia para melhor compreender os fenômenos epidêmicos. Os primeiros modelos de estudo de propagações epidêmicas baseavam-se na premissa de homogeneidade no risco de infecção. A equação 1 apresenta um modelo simples apresentado para o cálculo da reprodutibilidade basal $\left(\mathrm{R}_{0}\right)$ de uma doença, que pode ser interpretado como seu potencial para desenvolver-se de forma epidêmica. Considerando uma população não estruturada, obtem-se equações como: $R_{0}=c \times b \times D \times$ $S^{\prime} R_{0}{ }^{\prime}$ (CODEÇO; COELHO, 2008) é o indice de reprodutibilidade basal da doença (uma das formas de se medir o potencial epidêmico de uma doença), 'c' é o número de contatos com outras pessoas feitas por um individuo infectado em um dia, 'b' é a probabilidade de transmissão da doença por contato, ' $D$ ' é a duração da infecção e 's' é a proporção de individuos suscetiveis a serem infectados na população.

Nota-se que o modelo da equação captura os elementos mais fundamentais na determinação da propagação da epidemia, sendo incapaz de abranger situações mais complexas. Por exemplo, ao aplica-lo em populações reais que possuem formas de estruturação interna, como a formação de grupos ou redes sociais, as relações derivadas destas estruturas devem ser levadas em conta, sob a pena de gerar imprecisões nos resultados e conclusões obtidas. 
Esta questão conceitual deve-se a que os eventos que ocorrem nas populações estruturadas não podem ser tratados como eventos completamente independentes, como se faz em muitos estudos. De acordo com a definição para a dependência de eventos:

Ronald Ross, em 1916, chamou a atenção para a distinção entre "acontecimentos dependentes" e "independentes", em sua Teoria geral dos acontecimentos, colocando as doenças transmissíveis na primeira categoria. Acontecimentos dependentes seriam definidos pela dependência entre a ocorrência de novos eventos (incidência) e o número de eventos presentes (prevalência). Esta dependência surgiria do mecanismo contagioso subjacente ao processo de transmissão. Mas não apenas as doenças transmissíveis estariam nesta categoria, mas também eventos de outras naturezas - como comportamentos sociais que se propagam via troca de ideias e papéis entre pessoas. Rumores e modismos seriam exemplos de processos que emergiriam por este mecanismo. (CODEÇO; COELHO, 2008, p. 1768)

Sobre a complexidade e importância da análise das relações de dependência entre eventos para a modelagem de epidemias e sua ruptura com a "metodologia cientifica tradicional", vale notar que:

[...] a dependência demanda métodos de análise e de estudo distintos da metodologia cientifica tradicional, que está predominantemente baseada no conceito de observações independentes (grupos tratamento $\mathrm{x}$ controle, expostos x não expostos), cujos métodos de inferência são muitas vezes sensíveis à violação do pressuposto de independência. No entanto, o processo de transmissão por contágio cria fortes dependências entre eventos, mesmo entre indivíduos ligados apenas por longas cadeias de contato. Eventos dependentes implicam que efeitos indiretos das intervenções sempre surgirão - tratar ou intervir sobre um indivíduo afetará o risco de outro indivíduo; o risco do indivíduo sofrer um evento dependerá da coletividade, e a resposta do coletivo (epidemia ou não epidemia) dependerá não só das características pessoais, mas também da forma como as pessoas interagem entre si; e, por fim, as intervenções que realizamos (na forma de vacinação, tratamentos quimioterápicos e alterações de contato) afetam a própria população de microrganismos, que evoluem novas estratégias adaptativas (evolução de resistência é um exemplo). A relação individuo-população surge complexamente na epidemiologia das doenças transmissíveis. (CODEÇO; COELHO, 2008, p. 1768)

A questão da dependência dos eventos que estão associados à propagação de epidemias gera uma demanda por novos métodos e abordagens, dado que a "metodologia científica tradicional" não é capaz de fornecer o ferramental adequado para cenários onde haja dependência entre os eventos estudados. Uma proposta de caminho para buscar um método que responda a esse novo cenário é trazer os conceitos, ferramentais e métodos utilizados na 
análise de redes para, aliado ao ferramental matemático já existente e aos conhecimentos de outras disciplinas, complementar os trabalhos de análise de epidemias.

\section{As redes no estudo das epidemias}

O aperfeiçoamento dos modelos utilizado para descrever fenômenos epidêmicos, após o reconhecimento das relações de dependência que os afetam, demandou uma nova abordagem, uma que fosse capaz de modelar populações estruturadas em grupos. Uma das primeiras conceituações, proposta por Klovdahl, levava em conta a estrutura das populações humanas e seu caráter de rede:

Em 1985, Klovdahl propõe "a conceitualização da população como um conjunto de indivíduos ligados entre si formando uma grande rede". Esta abordagem proporcionaria um melhor entendimento do espalhamento de doenças infecciosas. Logo no início da epidemia de AIDS, ele pontuou que a extensão da transmissão da epidemia dependeria da estrutura das relações pessoais e que a compreensão destas estruturas seria útil para estimação do seu potencial epidêmico.(CODEÇO; COELHO, 2008, p. 1770)

Interpretar e analisar as populações humanas e suas complexas dinâmicas como redes de pessoas interagindo entre si, passa a ser uma forma de buscar incluir nos modelos variáveis antes não consideradas, e desenvolver métodos que garantam mais precisão no estudo dinâmica de epidemias. Uma alternativa, então, é voltar os estudos para as redes potenciais de risco:

O foco da maioria dos estudos está nas redes potenciais de risco, definidas como conjunto de indivíduos que compartilham comportamentos interacionais que "podem" servir de rotas para espalhamento do agente infeccioso. Isto é, elas existem independentemente da presença do agente infeccioso e, por isso, o adjetivo "potencial". No caso da AIDS e outras DST, sexo inseguro e/ou compartilhamento de drogas injetáveis seriam elementos de ligação entre pessoas; em doenças transmitidas pelo ar, como a gripe e a coqueluche, o compartilhamento de grandes ambientes fechados definiria as redes potenciais de risco.(CODEÇO \& COELHO, 2008, p. 1771)

Esta nova forma de abordagem propõe que os modelos levem em conta informações que contemplam e capturam a formação de potenciais estruturas de propagação epidêmicas nas populações, oriundas de "comportamentos interacionais", para gerar modelos mais precisos e fidedignos. As redes também são capazes de incorporar aos modelos informações de outras estruturas presentes na população, como as estruturas sociais:

Enquanto que o estudo das redes de risco tem recebido um olhar mecanistico, com ênfase na caracterização das estruturas, mecanismos e topologias que facilitam a difusão de doenças, outro conjunto de redes relevantes para a saúde, as redes sociais, tem sido alvo de um 
olhar mais fenomenológico (com ênfase na descrição de suas propriedades emergentes). As redes sociais, neste contexto, são definidas como estruturas sociais que influenciam a resposta dos indivíduos às infecções (seja nos comportamentos de risco e proteção, na capacidade de recuperação e resposta a tratamentos, na busca por serviços de saúde). As conexões entre elementos destas redes são definidas por laços de amizade, confiança e/ou poder de influência, e através delas percolam informações, comportamentos e cuidados à saúde. Estas redes interferem indiretamente no curso de uma epidemia, dado que sua estrutura influencia o apoio social recebido pelos indivíduos que a compõem. $\mathrm{O}$ apoio social que emerge destas redes é classificado em quatro tipos: emocional, instrumental ou material (como ajuda financeira ou física), informacional (conselhos, orientações), e de interação social positiva (pessoas com quem se divertir). (CODEÇO; COELHO, 2008, p. 1771)

Desta forma, modelar o potencial de determinada epidemia em uma população incorporando as redes de risco e as redes sociais que se formam em seu espaço de interação, permite um ganho de precisão nos modelos gerados. No entanto, mapear e entender estas redes não são tarefas simples, surgindo dificuldades a serem contornadas:

O primeiro desafio dos estudos de redes de transmissão surge, porém, no fato da metodologia estar fortemente baseada na análise de dados de redes completas (possível quando pequenos grupos são estudados), enquanto que dados sobre redes de contato na população seriam praticamente impossíveis de serem obtidos em sua totalidade. (CODEÇO; COELHO, 2008, p. 1771)

Esta dificuldade de desenvolver estudos com uma abrangência mais próxima do total da população, no entanto, tem sido contornada pelo uso de métodos de amostragem e análises matemáticas mais elaboradas, bem como através do uso das tecnologias de informação, tanto para captura, quanto para processamento de dados relativos às populações e computação dos modelos.

\section{Novas abordagens na modelagem de epidemias}

Apresentaremos aqui exemplos de formas mais complexas de modelar epidemias junto com uma breve explicação de como as mesmas podem colaborar para expandir o horizonte de análise dos modelos.

Autômatos celulares (AC), são uma modelagem proposta por John Von Neumann e Stanislaw Marcin Ulam para organismos auto reprodutores e crescimento celular que também pode ser usada com sucesso para modelar a propagação de epidemias, onde cada indivíduo ou conjunto de indivíduos, comporta-se como uma célula do autômato. A cerca dos AC's temos que "um AC é definido pela geometria da estrutura que contém as suas células, ou seja, pela 
geometria do seu reticulado; pelos estados permitidos para as células; pelas regras de transição entre esses estados; e pela vizinhança das células." (SCHIMIT, 2010, p. 22)

O uso dos autômatos permite inserir nos modelos aspectos geográficos e topológicos das populações, através da parametrização da geometria de seu reticulado; permite abordar de maneira diferente doenças com dinâmicas diferentes, a partir da definição dos estados permitidos e também compreender diferentes formas de interação entre os indivíduos através das regras de transição. Destaque para o fato de que as regras de transição entre os estados podem ainda ser probabilistas, o que confere um caráter de aleatoriedade que permite refinar ainda mais as previsões.

Além dos autômatos celulares, a tese de Schimit (2010) também apresenta modelos baseados em equações diferenciais ordinárias (EDO’s) em dois cenários: considerando o uso de vacinação e sem considerar o uso de vacinas. Além disso, Schimit (2010) discute o uso da Teoria do Jogos para melhor compreensão dos efeitos das políticas de vacinação na contenção de epidemias.

Vejamos exemplos de modelagem de epidemias que utilizam a teoria dos autômatos celulares:

- Boccara e Cheong (1993) mostram como doenças se propagam em um modelo SIS (suscetível-infectado-suscetível, característico de doenças onde a cura não confere ao indivíduo imunidade à doença), com movimentação de células;

- Ahmed e Agiza (1998) e Monteiro (2007) combinam o uso de autômatos celulares com equações diferenciais para analisar como o tempo de incubação (ou seja, o tempo em que o antígeno da doença permanece no hospedeiro sem desencadear a doença) influencia a propagação de uma doença;

- Kleczkowski e Grenfell (1999) e Watts e Strogatz (1998) utilizam o modelo SIR (suscetível-infectado-removido) para estudar a propagação de doenças em autômatos conectados por redes aleatórias;

- Fuentes \& Kuperman (1999), Mikler (2005) e Yakowitz (1990) analisam as influências da estrutura espacial da população na preservação de uma doença contagiosa;

- Monteiro e Chimara (2006) estudam a propagação de doenças em um autômato celular probabilista (ACP) bidimensional, onde considera-se que cada célula seja influenciada pelos 8 vizinhos mais próximos; 
- Monteiro (2006) modela um fenômeno neural conhecido como depressão alastrante (spreading depression), que consiste na propagação de uma onda de inatividade bioelétrica no cérebro usando um AC bidimensional;

- Schimit (2010) compara a modelagem através de AC e do uso de EDO's, analisa o efeito da remoção de conexões (que pode ser feita através do uso de máscaras ou da recomendação de isolamento temporário de indivíduos) para conter epidemias e, aplicando a Teoria dos Jogos, estuda a relação entre indivíduos suscetíveis e o governo frente a doenças contagiosas.

A lista de exemplos acima mostra como a modelagem conceitual da dinâmica de epidemias utilizando o conceito de redes de autômatos celulares e um ferramental matemático mais robusto, como é o caso das EDO's nos permitem contemplar uma gama maior de fatores que influenciam a propagação das epidemias, como é o caso em populações estruturadas.

\section{Conclusão}

O estudo da história do desenvolvimento de modelos para analisar a dinâmica de propagação de epidemias de doenças e outros fenômenos epidêmicos permite concluir que as populações humanas não são massas passivas e desestruturadas de pessoas, facilmente mapeáveis através de números agregados. Diferentes formas de estruturação social e culturas coexistem dentro de uma mesma população, o que resulta em um complexo mosaico de formas e possibilidades de interação, gerando diferentes padrões de comportamentos interacionais e, em também, diferentes suscetibilidades e dinâmicas para ocorrência de fenômenos epidêmicos. Desta forma, ferramentas conceituais que não consigam levar em conta esta essência dinâmica das populações humanas, estarão expostas ao risco de gerar previsões e modelos desviados da realidade social.

Por fim, mostramos como conceitos tais como o da estruturação de populações em redes podem e devem ser combinados com ferramentais de disciplinas como a matemática e a economia para possibilitar uma melhor compreensão, prevenção e combate das epidemias. 


\section{Referências Bibliográficas}

AHMED, E.; AGIZA, H.n.. On modeling epidemics Including latency, incubation and variable susceptibility. Physica A: Statistical Mechanics and its Applications, [s.1.], v. 253, n. 1-4, p.347-352, maio 1998. Elsevier BV.

BOCCARA, N; CHEONG, K. Critical behaviour of a probabilistic automata network SIS model for the spread of an infectious disease in a population of moving individuals. Journal Of Physics A: Mathematical and General, [s.1.], v. 26, n. 15, p.3707-3717, 7 ago. 1993. IOP Publishing.

CASTELLS, Manuel. A Sociedade em Rede. 6. ed. São Paulo: Paz e Terra, 2013.

CODEÇO, Cláudia Torres; COELHO, Flávio Codeço. Redes: um olhar sistêmico para a epidemiologia de doenças transmissíveis. Ciência \& Saúde Coletiva, [s.1.], v. 13, n. 6, p.1767-1774, 2008. FapUNIFESP (SciELO).

FUENTES, M.a.; KUPERMAN, M.n.. Cellular automata and epidemiological models with spatial dependence. Physica A: Statistical Mechanics and its Applications, [s.1.], v. 267, n. 3-4, p.471-486, 1999. Elsevier BV.

GRAHAM , S. \& MARVIN, S. Telecommunications and the City: Electronic Spaces, Urban Places. 1997. Londres: Routledge.

KLECZKOWSKI, Adam; GRENFELL, Bryan T.. Mean-field-type equations for spread of epidemics: the 'small world' model. Physica A: Statistical Mechanics and its Applications, [s.1.], v. 274, n. 1-2, p.355-360, 1999. Elsevier BV.

MIKLER, Armin R.; VENKATACHALAM, Sangeeta; ABBAS, Kaja. MODELING INFECTIOUS DISEASES USING GLOBAL STOCHASTIC CELLULAR AUTOMATA. Journal Of Biological Systems, [s.1.], v. 13, n. 04, p.421-439, 2005.

MICHAELIS. Moderno Dicionário da Língua Portuguesa. Disponível em: <http://michaelis.uol.com.br/moderno/portugues/index.php>. Acesso em: 10 jul. 2017.

MONTEIRO, L. \& CHIMARA, H. C. B. J. Big cities: Shelters for contagious diseases. Ecological Modelling, pp. 258-262, 2006.

MONTEIRO, L. H. A., SASSO, J. B. \& CHAUI BERLINCK, G. J. Continuous and discrete approaches to the epidemiology of viral spreading in populations taking into account the delay of incubation time. Ecological Modelling, Volume 201, pp. 553-557, 2007.

MONTEIRO, L. H. A.; PAIVA, D. C.; PIQUEIRA, J. R. C.. SPREADING DEPRESSION IN MAINLY LOCALLY CONNECTED CELLULAR AUTOMATON. Journal Of Biological Systems, [s.1.], v. 14, n. 04, p.617-629, dez. 2006. World Scientific Pub Co Pte Lt. 
NASA, 2015. NASA confirms evidence that liquid water flows on today's Mars. Disponível em: <https://www.nasa.gov/press-release/nasa-confirms-evidence-that-liquid-water-flows-ontoday-s-mars/>. Acesso em 28 de Setembro de 2015.

SCHIMIT, P. H. T.. Modelagem e controle de ropagação de epidemias usando autômatos celulares e teoria dos jogos. São Paulo. 2010.

WATTS, J. D. \& STROGATZ, S. H. Collective dynamics of 'small-world' networks. Nature, pp. 440-442, 1998.

YAKOWITZ, S. Cellular automaton modeling of epidemics. Applied Mathematics and Computation, , pp. 41-54, 1990. 